\title{
Dynamics of brain-derived proteins in cerebrospinal fluid
}

\author{
Hansotto Reiber* \\ Neurochemisches Labor der Neurologischen Klinik, Universität Göttingen, Robert-Koch-Str. 40, D-37075 Göttingen, Germany
}

Received 27 November 2000; received in revised form 18 May 2001; accepted 24 May 2001

\begin{abstract}
Background: The recent theory of blood-cerebrospinal fluid (CSF) barrier function and dysfunction connects molecular flux and CSF flow rate. A reduced CSF flow rate is sufficient to account for the observed hyperbolic relation between different blood-derived protein concentrations in CSF in cases of a blood-CSF barrier dysfunction. Methods: The dynamics of brain-derived proteins in CSF are investigated with reference to the CSF flow rate measured by CSF/serum albumin concentration quotient. Results: Proteins from neurons or glial cells, tau protein, neuron-specific enolase, S-100 protein, all enter CSF primarily in the ventricular and cisternal space. Their concentration between normal ventricular and lumbar CSF is decreasing (in contrast to blood-derived proteins), and in the case of pathologically decreasing CSF flow rate, the concentration in lumbar CSF remains invariantly constant. Concentrations of the primarily leptomeningeal proteins, $\beta$-trace protein and cystatin $\mathrm{C}$, increase between normal ventricular and lumbar CSF, and in the case of pathologically decreased CSF flow rate they increase linearly in lumbar CSF (concentrations of blood-derived proteins increase non-linearly). Conclusions: A satisfactory physiological explanation can now be given for the dynamics of proteins in CSF consisting of both brain- and blood-derived fractions (transthyretin, soluble intercellular adhesion molecule (s-ICAM)), as well as the disputed decrease of leptomeningeal protein concentrations ( $\beta$-trace protein, cystatin $C$ ) in cases of bacterial meningitis is also explained. The biophysical treatment of dynamics in the ventricular and lumbar CSF extends the new theory and shows that CSF flow rate is the most relevant parameter for understanding the pathological changes of both blood- and brain-derived proteins in CSF. The impact on diagnosis of neuro-degenerative diseases is discussed. (C) 2001 Elsevier Science B.V. All rights reserved.
\end{abstract}

Keywords: Cerebrospinal fluid (CSF); CSF flow; Blood-CSF barrier function; Tau protein; S-100 protein; Neuron-specific enolase (NSE); $\beta$-trace protein (prostaglandin D synthase); Cystatin C; Transthyretin; Soluble intercellular adhesion molecule (s-ICAM); Carcinoembryonic antigen (CEA)

\section{Introduction}

The increasing diagnostic relevance of brain-derived proteins in cerebrospinal fluid (CSF) led to the awareness that their particular dynamics in CSF [1]

\footnotetext{
* Tel.: +49-551-39-66-19; fax: +49-551-39-20-28.

E-mail address: hreiber@med.uni-goettingen.de (H. Reiber).
}

are different from the dynamics of blood-derived proteins [2,3]. So far, there have been no attempts to describe the general dynamics of brain proteins in CSF. With the recognition of the particular relevance of CSF flow rate modulating the concentrations of blood-derived proteins in CSF of patients with a blood-CSF barrier dysfunction, it is of theoretical and diagnostic relevance [4-6] to investigate the 
impact of CSF flow rate on CSF concentrations for brain-derived proteins. The relevance of CSF flow was discussed circa 1700 by Fantony and in 1875 by Key and Retzius (references in Ref. [7]) and achieved scientific relevance by an experimentally founded physiology of CSF flow [8]. Current imaging techniques, such as nuclear magnetic resonance spectroscopy (references in Refs. [9,10]), give direct evidence for changing CSF flow rate in neurological diseases $[9,10]$. The CSF velocity rate of children from 1 to 17 years, measured by MRI, are on an average higher than the rate values in adults (Ref. [9] and reference therein). These relations correspond to the lower CSF protein concentration in younger age and an increasing protein concentration with decreasing CSF flow rate in older age. The influence of CSF flow rate on protein concentrations in CSF has been described in earlier reports by linear approaches [11,12].

The new theory that combines molecular flux and CSF flow rate [2], shows CSF flow rate as the main modulator of pathological CSF protein concentrations, i.e., reduced CSF flow rate is sufficient to explain quantitatively the empirically measured, non-linear increase of blood-derived CSF protein concentrations in neurological diseases. Potentially, also the view of blood-CSF barrier dysfunction could be changed from the still widespread understanding of a morphological "leakage"-model to a biophysical concept of increased molecular flux with decreasing CSF flow rate $[2,6]$.

\subsection{Blood-derived proteins in CSF}

The main fraction of proteins in the normal cerebrospinal fluid (CSF) originates from blood, e.g., albumin which constitutes $35-80 \%$ of total protein in CSF [6]. In neurological diseases with a bloodCSF barrier dysfunction, all blood-derived protein fractions in CSF are increased. This increased transfer of blood proteins into CSF is characterized by a hyperbolic function between protein concentrations in CSF. The hyperbolic discrimination function for the upper limit of the reference range for blood-derived fractions in CSF is the basis for established CSF serum quotient diagrams, now of widespread use in the diagnosis of neurological diseases $[4-6,13]$.

\subsection{Brain-derived proteins in CSF}

About $20 \%$ of the proteins in CSF are predominantly brain-derived, but rarely brain-specific [14] The basic feature of predominantly brain-derived proteins is their higher concentration in CSF compared to serum, inducing a net flux out of CSF compared with blood proteins with a net flux into CSF (Table 1). For some of the predominantly brain-derived proteins, a blood-derived fraction in CSF is not negligible.

Together with the pathological changes of CSF concentrations of blood-derived proteins, a particular modulation of the concentrations of brain-derived

Table 1

CSF concentration and concentration gradients for blood- and brain-derived proteins in lumbar CSF with calculated intrathecal fraction (IF)

\begin{tabular}{|c|c|c|c|c|}
\hline Proteins $^{\mathrm{a}}$ & $\begin{array}{l}\text { MW } \\
(\mathrm{kDa})\end{array}$ & $\begin{array}{l}\text { CSF } \\
\text { concentration }\end{array}$ & $\begin{array}{l}\mathrm{CSF} / \\
\text { serum ratio }\end{array}$ & $\operatorname{IF}(\%)^{\mathrm{b}}$ \\
\hline$\beta$-Trace-Prot. & 25 & $16.6 \mathrm{mg} / 1$ & $34: 1$ & $>99$ \\
\hline Cystatin C & 13.3 & $3.1 \mathrm{mg} / 1$ & $5: 1$ & $>99$ \\
\hline Tau-Protein & $55-74$ & $0.20 \mu \mathrm{g} / 1$ & $10: 1^{c}$ & $>99$ \\
\hline S-100 B & 21 & $1.5 \mu \mathrm{g} / 1$ & $18: 1$ & $>99$ \\
\hline NSE & 78 & $8 \mathrm{mg} / 1$ & $1: 1$ & $>99$ \\
\hline Transthyretin & $55^{\mathrm{d}}$ & $17 \mathrm{mg} / 1$ & $1: 18$ & $\sim 90$ \\
\hline $\mathrm{ACE}^{\mathrm{e}}$ & 150 & - & $1: 100$ & $\sim 65$ \\
\hline s-ICAM & 90 & $1.5 \mu \mathrm{g} / 1$ & $1: 190$ & $\sim 30$ \\
\hline Albumin & 67 & $245 \mathrm{mg} / 1$ & $1: 205^{\mathrm{f}}$ & 0 \\
\hline IgG & 150 & $25 \mathrm{mg} / 1$ & $1: 440^{\mathrm{f}}$ & 0 \\
\hline $\operatorname{IgA}$ & 170 & $1.0 \mathrm{mg} / 1$ & $1: 800^{\mathrm{f}}$ & 0 \\
\hline $\operatorname{IgM}$ & 900 & $0.2 \mathrm{mg} / 1$ & $1: 3400^{\mathrm{f}}$ & 0 \\
\hline
\end{tabular}

${ }^{\text {a }}$ Source of data is indicated in Material and methods.

${ }^{\mathrm{b}} \mathrm{IF}=$ intrathecal fractions, are calculated as shown in Material and methods and Ref. [1].

${ }^{\mathrm{c}}$ Calculated with reference to median of serum concentration $20 \mathrm{ng} / \mathrm{l}$ ( $n=60$ normal blood donors); the value confirms Ref. [16]. The tau protein concentrations in blood vary extremely between $<10$ and $>1000 \mathrm{ng} /$.

${ }^{\mathrm{d}}$ Transthyretin $(54 \mathrm{kDa})$ passes from blood into CSF associated with the retinol-binding protein $(21 \mathrm{kDa})$. Data represent the mean from $n=27$ control patients.

${ }^{\mathrm{e}}$ For angiotensin converting enzyme (ACE), the CSF/serum ratio refers to measured total activities instead of concentrations. The molecular weight refers to the main fraction [1]. Data originate from Ref. [1].

${ }^{\mathrm{f}}$ The ratios refer to mean quotients $\left(Q_{\mathrm{IgG}}=2.28 \times 10^{-3}, Q_{\mathrm{IgA}}\right.$ $=1.26$ and $\left.Q_{\mathrm{IgM}}=0.295 \times 10^{-3}\right)$ calculated with the hyperbolic functions for mean $Q_{\mathrm{Alb}}=4.9 \times 10^{-3}$ [2]. 
proteins is observed [1], depending on the source of the proteins. So far, a theoretical approach for understanding the dynamics of brain proteins has been missing, as has a corresponding evaluation concept for brain proteins to facilitate the diagnosis of neurological diseases. The analysis of brain proteins represents an important extension of the basic CSF analysis program. As an example of the increasing diagnostic relevance, brain proteins help to differentiate between different causes of dementia, like Alzheimer's disease, Creutzfeldt-Jakob disease or multiinfarct dementia [15-18].

This paper describes the empirical data for brain proteins with changing CSF flow rate and gives for the first time a theoretically founded, physiologically relevant interpretation of the dynamics of brain proteins in CSF and points to the consequences for clinically relevant data evaluation.

\subsection{Different sources of brain-derived proteins}

Three groups of brain-derived proteins investigated in this study are selected as representatives of different origins and dynamics in CSF: (a) proteins originating from neurons and glial cells, like tau protein, S-100 and neuron-specific enolase, which are primarily released into ventricular and cisternal $\mathrm{CSF}$, (b) proteins which are primarily released from leptomeninges into CSF, like $\beta$-trace protein and cystatin $\mathrm{C}$ and (c) proteins with a non-negligible blood-derived fraction in CSF, like transthyretin, angiotensin converting enzyme (ACE) or the soluble intercellular adhesion molecule (s-ICAM) as a third group.

Neuron-specific enolase (NSE), the gamma, gamma dimer with a molecular weight of $78 \mathrm{kDa}$ originates predominantly from neurons and neuroendocrine cells. The large amount found in the brain means that NSE is a very sensitive marker for fast neuronal degradation. It can be detected either in CSF [19,20], e.g., in Creutzfeldt-Jakob disease [17], or, in blood [21] for the prognosis of clinical outcomes after cerebral hypoxia, brain infarction or head/brain trauma.

S-100 protein is an acidic, calcium-binding protein found in the brain as homodimer or heterodimer with a molecular weight of $21 \mathrm{kDa}$. S-100B, the beta, beta dimer, is present in high concentrations in glial cells and Schwann cells. Tissue-specific isoforms are also found in non-nervous tissue. The biological function of the protein is not yet clear. Due to its increased release into CSF and serum after cell degradation in CNS, S-100B has diagnostic relevance $[18,22]$, similar to NSE.

Tau proteins (MW 55 to $74 \mathrm{kDa}$ ) modulate assembly and stability in axonal and dendritic microtubules of neurons, but are also found in astrocytes and oligodendrocytes. The hyperphosphorylated form of tau protein, associated with paired helical filaments (PHF-tau), is increased in CSF of patients with Alzheimer's disease and has diagnostic relevance in combination with decreased $\beta$-amyloid protein concentration $[15,16]$.

$\beta$-trace protein (prostaglandin D synthase) is a small secretory protein $(\mathrm{MW}=25 \mathrm{kDa})$. The biological function of $\beta$-trace protein remains unknown in spite of the detected prostaglandin $\mathrm{D}$ synthase activity [23-25]. Diagnostic relevance of $\beta$-trace protein comes from its use as a marker to detect CSF in nasal secretions from patients with rhinoliquorrhea. $\beta$-Trace protein in cerebrospinal fluid originates exclusively from the brain. The main sources of biosynthesis in the brain for this protein are the leptomeninges [24-26].

Cystatin $\mathrm{C}$, the former gamma trace protein, is a cysteine proteinase inhibitor with a molecular weight of $13.3 \mathrm{kDa}$. It is produced by all nucleated cells, including neuroendocrine cells and cortical neurons [27]. A higher concentration of cystatin $\mathrm{C}$ in CSF than in serum allows cystatin $\mathrm{C}$ to be classified as a predominantly brain-derived protein in CSF with a negligible blood-derived fraction $(<1 \%)$. Besides a dominant release from the choroid plexus compared to the residual brain [28], a release of cystatin C from leptomeningeal cells in vitro has been reported [29].

Transthyretin, the former prealbumin, functions as a carrier protein for thyroxine and for vitamin A by one-to-one association with retinol binding protein [30]. Transthyretin in CSF originates predominantly from the choroid plexus as the only source of synthesis in brain cells [31]. In contrast to NSE, S-100B, tau protein and $\beta$-trace protein, the blood-derived concentration of transthyretin is not negligible. With an 18-fold higher concentration of liver-derived transthyretin in blood compared to CSF, a small blood-derived fraction of about $10 \%$ can be calcu- 
lated to contribute to transthyretin concentration in normal lumbar CSF [1]. Large sets of transthyretin data for ventricular, cisternal or lumbar CSF have been reported earlier [32], indicating that there is a small decrease of concentration between ventricular and lumbar CSF and an increase of transthyretin concentrations with reduced CSF turnover below a spinal blockade with a restricted CSF flow rate.

Soluble intercellular adhesion molecule (s-ICAM) in CSF [33] has a blood-derived fraction of 70\% and a brain-derived fraction of $30 \%$. It is of particular interest due to its metabolic interferences in pathological conditions [34]. There is no actual clinical relevance for analysis.

Angiotensin converting enzyme has restricted clinical relevance for diagnosis of neurosarcoidosis. Its CSF concentration is primarily blood-derived, and particular care is needed to detect its increased brain-derived fraction in case of blood-CSF barrier dysfunctions [1].

\section{Material and methods}

The methods for analysis of $\beta$-trace protein [26], transthyretin [1], S-100 [18], NSE [17,21], tau protein [16], s-ICAM [33] and ACE [1] are performed as reported in the references. Only for tau protein in serum the enzyme immunoassay (Innogenetics, The Netherlands) was modified for higher sensitivity by increasing the sample volume threefold and reducing the reagent volume to $1 / 3$ with the threefold concentration of its constituents. Cystatin $\mathrm{C}$ is analysed with a particle amplified nephelometric assay (Dade Behring, Marburg, Germany). Albumin, IgG, IgA and $\operatorname{IgM}$ are analysed by immunochemical nephelometry [4].

Intrathecal fractions (IF), as shown in Fig. 1 for $\mathrm{IgG}$, are calculated as reported for $\mathrm{IgG}, \operatorname{IgA}, \operatorname{IgM}$ $[2,6]$ with reference to the upper discrimination line $\left(Q_{\mathrm{Lim}}\right)$ of the reference range for blood-derived protein fraction in CSF: $\mathrm{IgG}_{\mathrm{IF}}=\mathrm{IgG}_{\mathrm{Loc}} / \mathrm{IgG}_{\mathrm{CSF}} \times 100$ $=\left(1-Q_{\mathrm{Lim}} / Q_{\mathrm{IgG}}\right) \times 100$ in [\%] of total CSF concentration; with $Q_{\mathrm{Lim}}=a / b \sqrt{\left(Q_{\mathrm{Alb}}\right)^{2}+b^{2}}-c$, the hyperbolic function, in which the values $a / b ; b^{2}$, and $c$ are molecular size related, empirical values $[2,6]$. From the calculated intrathecal fractions in

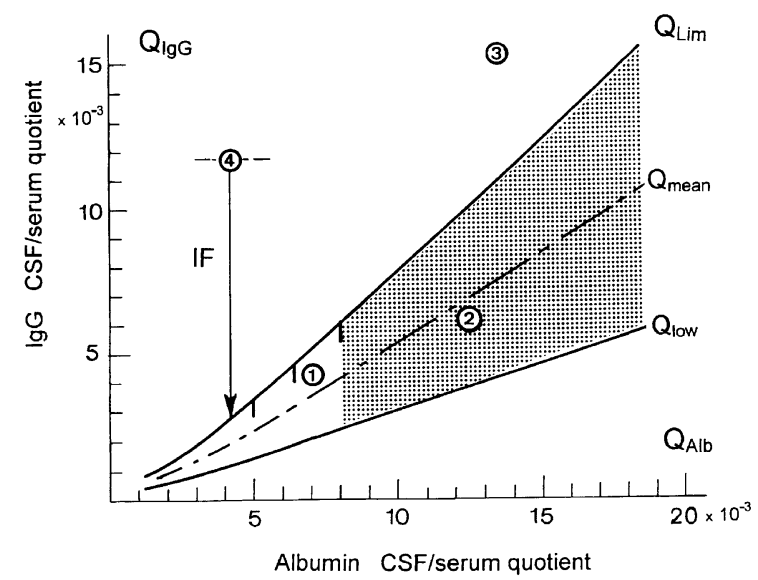

Fig. 1. CSF/serum quotient diagram for $\operatorname{IgG}$ with hyperbolic reference range, according to Reiber [2]. The reference range for the blood-derived fraction of $\mathrm{IgG}$ in CSF was characterized from data of 4300 patients [2], which had no intrathecal IgG synthesis (no oligoclonal $\mathrm{IgG}$ in CSF). The patients had either a normal blood-CSF barrier function $\left(Q_{\text {Alb }}\right.$ values in range 1$)$ or a bloodCSF barrier dysfunction (range 2). The diagram of the CSF/serum quotients of $\mathrm{IgG}$ and albumin represents the upper border line ( $\left.Q_{\mathrm{Lim}}\right)$, the mean $\left(Q_{\text {mean }}\right)$ and the lower border line $\left(Q_{\text {low }}\right)$ of the reference range. These lines are empirically fitted and theoretically founded hyperbolic functions [2], see legend of Fig. 2. $Q_{\text {Lim }}$ represents the hyperbolic function which can discriminate the blood-derived $\operatorname{IgG}$ fraction (below $Q_{\mathrm{Lim}}$ ) and a pathological brain-derived, intrathecal fraction (IF, above $Q_{\mathrm{Lim}}$ ). Albumin $\mathrm{CSF} /$ serum quotients, $Q_{\mathrm{Alb}}$, larger than the age-related reference value (vertical bars shown for patients up to 15,40 or 60 years) indicate a blood-CSF barrier dysfunction, i.e., decreased CSF flow rate. The four main interpretation ranges (indicated by figures) are: range $1=$ normal $\mathrm{CSF}$ data, range $2=$ blood-CSF barrier dysfunction (dotted range), range $3=$ combined blood-CSF barrier dysfunction together with an intrathecal $\operatorname{IgG}$ synthesis; range $4=$ intrathecal IgG synthesis only. This figure represents a fraction of the complete diagrams [2]. For clinical practice purpose, the diagrams for $\operatorname{IgG}, \operatorname{IgA}, \operatorname{IgM}$ are used with logarithmic scales [6,39], also known as Reibergrams [6,13].

CSF, subsequently the residual blood-derived fractions in CSF are obtained. In cases where already under normal conditions a brain-derived fraction is present, the normal, molecular size related gradient between blood and CSF is not directly detectable. The theoretical gradient for the purely blood-derived molecule is interpolated from known gradients of other molecules, in particular albumin (MW $67 \mathrm{kDa}$ ) with CSF $/$ serum $=1: 200$ and $\operatorname{IgG}(\mathrm{MW}=150 \mathrm{kDa})$ with CSF/serum 1:450. For a blood-derived 
molecule with a molecular size corresponding to 20-30 kDa, a CSF/serum ratio with about 1:30 was found (unpublished data). For S-100 (MW = 21 $\mathrm{kDa}$ ), such a value could be used to calculate the theoretical blood-derived fraction in the dominantly brain-derived CSF concentration. With the known mean serum concentration value and the calculated gradient, a mean blood-derived CSF concentration can be calculated and expressed as \% of the measured total CSF concentration. Calculations of blood-derived fractions in CSF have been reported for transthyretin [1], s-ICAM [33] and for ACE [1].

\subsection{Patients}

Lumbar CSF and serum samples originate from patients of the Department of Neurology, University Göttingen. All samples were taken for routine analysis with the informed consent of the patients. The presented data in the study are partly reevaluated from earlier publications of the Neurochemistry Laboratory in Göttingen.

In particular, this refers to Albumin, $\operatorname{IgG}, \operatorname{IgA}$ and IgM [2] for Figs. 1 and 2; prothrombin [35] for Fig. 2 ; intercellular adhesion molecule-1 (s-ICAM-1) [33] and $\beta$-trace protein [36] for Fig. 3. The data for angiotensin converting enzyme originate from reference [1]. The mean values of carcinoembryonic antigen in Fig. 2 refer to unpublished data from $n=60$ non-tumour patients.

The already published data as well as the new data in this article are based on the following diagnostic criteria.

(1) Normal control patients have been defined clinically (absence of brain-organic diseases according to clinical and imaging criteria) and via their CSF and blood data (normal CSF leucocyte count and protein values, and normal blood leucocytes and plasma C-reactive protein (CRP) values). Single subgroups of patients, e.g., with tension headache or non-inflammatory polyneuropathies, as far as they have been found to be indistinguishable in mean and range of concentration values from the normal reference range, have been combined for statistical evaluation.

(2) Non-inflammatory diseases with blood-CSF barrier dysfunction: This group contained patients with increased albumin CSF/serum quotients due to

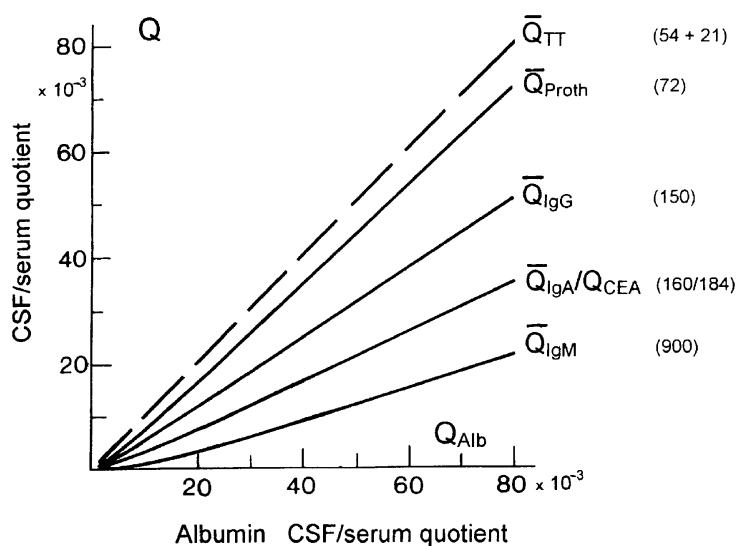

Fig. 2. Blood-derived proteins and blood-CSF barrier dysfunction. The hyperbolic curves show the mean CSF/serum quotients, $\bar{Q}$, of blood-derived proteins with different molecular size as a function of decreasing CSF flow rate [2]. The decreasing flow rate or increasing blood-CSF barrier dysfunction is indicated by the increasing albumin CSF/serum quotient, $Q_{\mathrm{Alb}}$. The theoretical, calculated blood-derived fraction of transthyretin (TT) is shown by the dashed line with $45^{\circ}$ (TT passes the barrier associated with the retinol binding protein [1] and has therefore a size similar to albumin). Empirical mean quotients for prothrombin (Proth.) [35], carcinoembryonic antigen (CEA) (Reiber, unpublished) and immunoglobulins IgG, IgA, IgM [2] are shown together with their molecular weights $(\mathrm{kDa})$. Hyperbolic functions are a consequence of non-linear interaction of molecular flux with CSF flow rate as derived from the laws of diffusion [2] with the general equation: $Q_{\mathrm{IgG}}=\left(\operatorname{erfc} z\left(D_{\mathrm{IgG}}: D_{\mathrm{Alb}}\right)^{0.5} / \mathrm{erfc} z\right) \times Q_{\mathrm{Alb}}$. This function shows that the relation depends only on the ratio of diffusion coefficients $\left(D_{\mathrm{IgG}}: D_{\mathrm{Alb}}\right)$, i.e., on the different size of the molecules. The hyperbolic functions for the single proteins are characterized empirically by three variables $(a, b, c)$ in a more usual form of the hyperbolic function, $Q_{\mathrm{IgG}}=a / b \sqrt{\left(Q_{\mathrm{Alb}}\right)^{2}+b^{2}}-c$.

spinal canal stenosis, a spinal tumour or disc prolapse. They showed increased CSF total protein and albumin quotients, normal cell counts as well as typical findings in electromyography and magnetic resonance tomography of the spine. Oligoclonal $\mathrm{IgG}$ in CSF was an exclusion criteria.

\subsection{Ventricular CSF}

Ventricular CSF samples originated from patients of the Neurosurgery Department of the University of Göttingen, routinely analysed to exclude a postsurgery inflammation. The samples have been extracted from a drainage. 


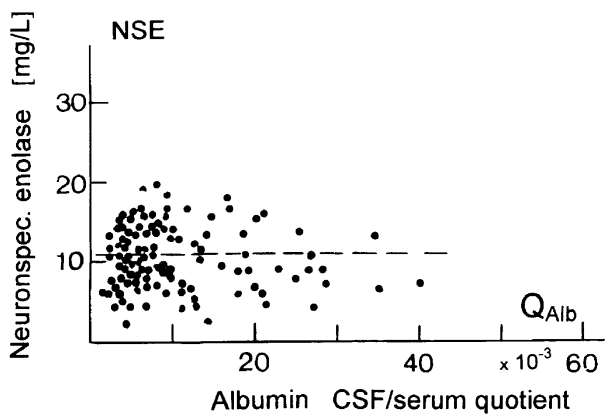

a

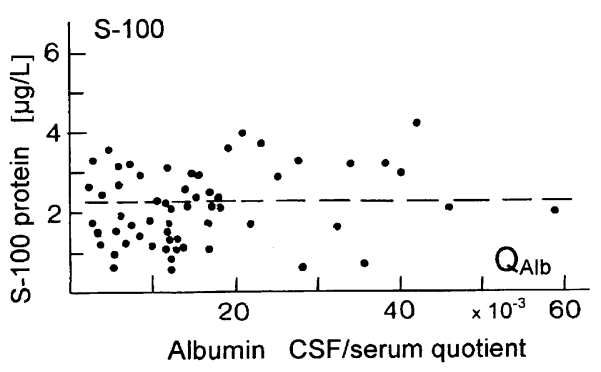

b
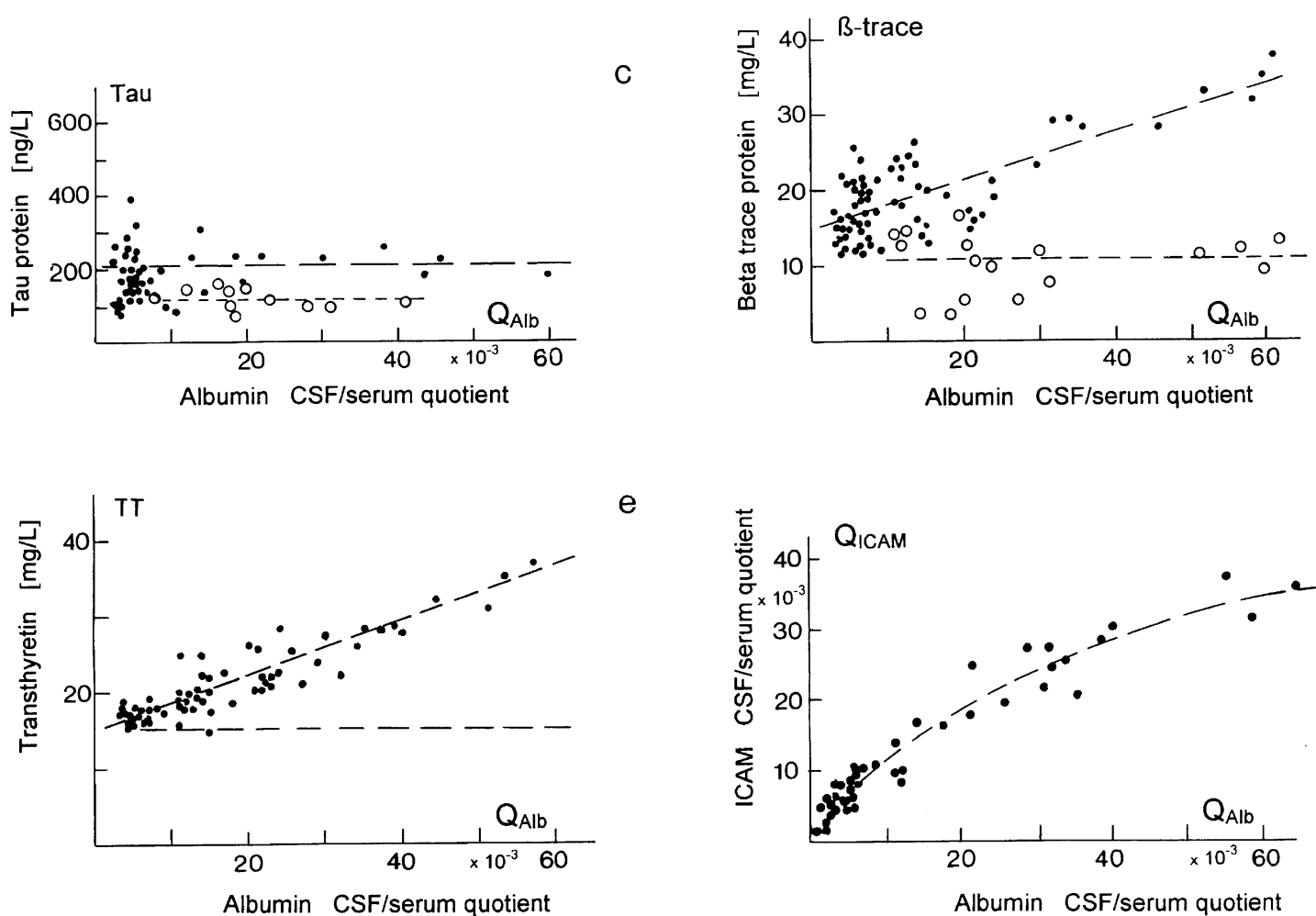

e

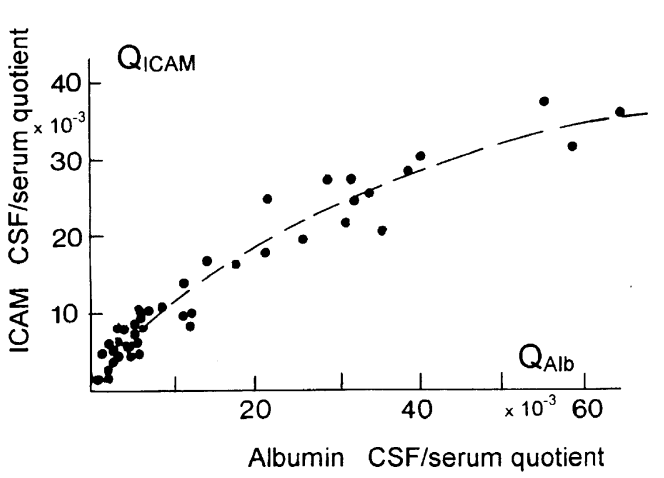

Fig. 3. Brain-derived proteins in CSF. (a) NSE concentration (mg/l) in lumbar CSF as function of the Albumin CSF/serum concentration quotient, $Q_{\mathrm{Alb}}$. For the regression line, a slope $=0$ was calculated. The data originate from normal control patients and patients with blood-CSF barrier dysfunctions. The patients with degenerative diseases, hypoxia or stroke have been excluded from this group. (b) S-100B protein concentration $(\mu \mathrm{g} / 1)$ in lumbar CSF as function of the albumin quotient, $Q_{\mathrm{Alb}}$. For the regression line, a slope $=0$ was calculated. The patients in this group fulfilled the same diagnostic criteria as patients in (a). (c) Tau protein concentration (ng/l) in lumbar CSF from patients with normal CSF, and non-inflammatory diseases with increased albumin quotients $(\mathbf{)})$. Cases of neuroborreliosis $(\bigcirc)$ are shown with a dashed regression line (slope $=0$ ). Diagnostic criteria are given in Section 2 and in [34] for neuroborreliosis. (d) Beta trace protein concentration (mg/l) in lumbar CSF from normal controls and patients with spinal canal stenosis $(\mathbf{)})$. The function of the regression line is shown in Eq. (2) in the text. Data of patients with a bacterial meningitis $(O)$ show lower values. The dashed regression line has a slope $=0$. The data are reevaluated from earlier reports $[26,36]$. (e) Total transthyretin concentration (mg/l) in lumbar CSF of normal controls and cases with blood-CSF barrier dysfunction ( ). The dashed line represents the calculated [1] mean concentration of choroid plexus derived transthyretin fraction in CSF. The data have been reported in Ref. [1]. (f) CSF/serum concentration quotients of the soluble intercellular cell adhesion molecule, $Q_{\mathrm{ICAM}}$, in lumbar CSF. Data are obtained from normal controls and patients with non-inflammatory diseases, as reported in Ref. [33]. The dashed curve represents the mean of the reference range. 
The group of "normal" ventricular CSF samples is recruited from a larger collection of samples with low albumin quotient, absence of haemoglobin or blood contamination as well as absence of oligoclonal IgG. The cell count was $<5 / \mu l$.

The group with increased ventricular albumin quotients was recruited from patient with a CSF flow restricting stenosis or tumour. For some parameters, the early bacterial infection (before onset of the humoral immune response) could be included.

\section{Results}

\subsection{CSF to serum gradient of brain-derived proteins}

The concentrations of predominantly brain-derived proteins in normal lumbar CSF together with the corresponding serum concentrations are shown in Table 1. We find large $\mathrm{CSF} /$ serum concentration ratios between 1:1 (NSE) and 34:1 ( $\beta$-trace protein) compared to 1:205 (albumin) or 1:3400 (IgM) for blood-derived proteins in CSF. The calculation of the brain-derived intrathecal fraction (IF) is $>99 \%$ for tau protein, NSE, S-100B, cystatin C or beta trace protein, but $0 \%$ for the proteins which, under normal conditions, are exclusively blood-derived. Brain-derived proteins with a non-negligible blood-derived fraction in CSF, like transthyretin, ACE or s-ICAM have intermediate CSF/serum ratios (1:18 to $1: 190)$ with intrathecal fractions between about $90 \%$ and $30 \%$, correspondingly.

\subsection{Ventricular to lumbar CSF concentration gradi- ent}

The concentration gradient between ventricular and lumbar CSF, as a second feature of brain-derived proteins, is shown in Table 2. Proteins released from brain cells (NSE, S-100B and tau protein) have the highest concentration in ventricular CSF decreasing along its flow path through the subarachnoid space. Vice versa, proteins released from leptomeninges into CSF are increasing in concentration along the subarachnoid space up to 11-fold (beta trace protein). The gradient for transthyretin refers to the choroid plexus derived fraction in CSF calculated from total transthyretin in CSF by subtraction of the blood-derived fraction (Section 2). The concentration of total transthyretin is decreasing slightly between ventricular and lumbar CSF, in spite of the small but steady net flux of blood-derived molecules into CSF, as consequence of the overall blood/CSF gradient shown in Table 1 . These data are consistent with an earlier report [32].

\subsection{Brain proteins and CSF flow rate}

A third feature of brain-derived proteins in CSF is their dynamics due to changing CSF flow rate in cases of neurological diseases with "blood-CSF barrier dysfunction". With pathologically decreasing CSF flow rate, i.e., increasing $Q_{\text {Alb }}$ in Fig. 3a-f, we find again differences between the first group of brain cell-derived proteins with constant concentrations, which show an invariance for changes of CSF flow rate, and the second group of leptomeningeal cell-derived proteins, which show a linearly increasing concentration. Data for the first group (tau protein, S-100B and NSE), and for the second group ( $\beta$-trace protein) are shown in the corresponding Fig. $3 a-d$. Proteins with non-negligible blood-derived fractions in addition to brain-derived fractions like transthyretin (Fig. 3e), soluble intercellular adhesion

Table 2

Concentration gradients of brain-derived proteins between normal ventricular (V) and lumbar (L) CSF For comparison, the corresponding data of the blood-derived protein albumin are shown, too.

\begin{tabular}{llllllll}
\hline & $\begin{array}{l}\text { NSE } \\
(\mathrm{ng} / \mathrm{ml})\end{array}$ & $\begin{array}{l}\text { S-100 B } \\
(\mathrm{ng} / \mathrm{ml})\end{array}$ & $\begin{array}{l}\text { Tau-Prot. } \\
(\mathrm{ng} / \mathrm{ml})\end{array}$ & $\begin{array}{l}\text { TT (ch. pl. }) \\
(\mathrm{ng} / \mathrm{ml})\end{array}$ & $\begin{array}{l}\beta-\text { Trace } \\
(\mathrm{mg} / \mathrm{l})\end{array}$ & $\begin{array}{l}\text { Cys. C } \\
(\mathrm{mg} / \mathrm{l})\end{array}$ & $\begin{array}{l}\text { Alb } \\
(\mathrm{mg} / \mathrm{l})\end{array}$ \\
\hline V-CSF & 22 & 5.3 & 0.32 & 17.0 & 1.5 & 0.87 & 100 \\
L-CSF & 10.8 & 1.5 & 0.21 & 15.3 & 16.6 & 3.1 & 245 \\
Gradient V:L & $2: 1$ & $3.5: 1$ & $1.5: 1$ & $1.1: 1$ & $1: 11$ & $1: 3.5$ & $1: 2.5$
\end{tabular}

$\mathrm{NSE}=$ Neuron-specific enolase; S-100B $=\beta, \beta$-dimer of S-100; Tau-Prot. $=$ total tau protein including PHF-tau; TT (ch. pl.) $=$ calculated tranthyretin fractions originating from choroid plexus (calculated blood-derived fraction substrate from total CSF concentration); $\beta$-Trace $=$ $\beta$-trace protein (prostaglandin D-synthase); Cys. $\mathrm{C}=$ cystatin $\mathrm{C}$ or former gamma trace protein; Alb = albumin. The mean values of data from ventricular CSF refer to groups of 7 to 13 patients (diagnosis as defined in Material and methods). 
molecule (s-ICAM, Fig. 3f) as well as ACE [1] represent a third group. The data for the source-related differences in the dynamics can be summarized for each protein.

\subsubsection{Neuron-specific enolase (NSE)}

With a CSF/serum concentration ratio of $1: 1$ (Table 1), the intrathecal (brain-derived) fraction of NSE in CSF can be calculated to be still $>99 \%$ (the blood-derived fraction is smaller than $1 \%$ due to an interpolated molecular size-dependent CSF/blood gradient of about 1:200 for a theoretical, solely blood-derived fraction). The concentration decreases between ventricular and lumbar CSF (2:1, Table 2). The mean NSE concentration in lumbar CSF does not change with increasing $Q_{\mathrm{Alb}}$ in case of a bloodCSF barrier dysfunction as shown in Fig. 3a, i.e., is invariant to reduced CSF flow rate.

\subsubsection{S-100 protein}

Table 1 shows that the brain-derived fraction in CSF for S-100B protein (beta, beta dimer) exceeds 99\% due to an empirical CSF/serum ratio of 18:1 and a theoretical molecular size-dependent blood-derived fraction $<1: 50$ (Section 2). Between ventricular CSF and lumbar CSF, the S-100 concentration decreases (3.5:1, Table 2). Fig. 3b shows the mean $\mathrm{S}-100$ concentration which is invariant to increasing $Q_{\mathrm{Alb}}$.

\subsubsection{Tau protein}

The CSF data in this paper refer to total tau protein including PHF-tau, the hyperphosphorylated form of tau protein. The lumbar CSF concentration exceeds 10-fold the blood concentration (Table 1) and tau protein concentration decreases between ventricular and lumbar CSF (Table 2). In cases of non-inflammatory CSF flow reduction, there is no increase of tau protein concentration in CSF with increasing $Q_{\mathrm{Alb}}$ (Fig. 3c). The reported lower values of CSF-tau protein in some inflammatory processes [16] are confirmed in Fig. 3c for cases of neuroborreliosis. The mean of decreased values was about $50 \%$ of normal lumbar CSF but again was independent of $Q_{\mathrm{Alb}}$, i.e., invariant to changing CSF flow rate.

\subsection{4. $\beta$-Trace protein}

$\beta$-trace protein in cerebrospinal fluid originates exclusively from the brain. The lumbar CSF concen- tration exceeds the blood concentration 34-fold (Table 1). In the case of increasing $Q_{\mathrm{Alb}}$ due to reduced CSF flow rate in non-inflammatory diseases (e.g., lumbar canal stenosis), the $\beta$-trace protein concentration increases linearly (Fig. 3d). A very particular influence, reducing lumbar CSF $\beta$-trace protein concentrations to about $60 \%$ of normal, is observed in bacterial or tuberculous meningitis [36] (Fig. 3d), but not in viral infections (e.g., HSV encephalitis) of the central nervous system. Amazingly, in this particular type of pathological processes the $\beta$-trace protein CSF concentrations in lumbar CSF are also independent of $Q_{\mathrm{Alb}}$, like NSE, S-100, or tau protein concentrations usually are.

\subsubsection{Cystatin $C$}

Cystatin C, also known as gamma-trace protein, has a fivefold higher concentration in CSF than in serum (Table 1), which allows us to speak of cystatin $\mathrm{C}$ as a brain-derived protein in CSF with a negligible blood-derived fraction $(<1 \%)$. As shown in Table 2, the concentration is increasing 3.5-fold between ventricular and lumbar CSF due to its leptomeningeal release. This normal rostro-caudal gradient is less steep $(30 \%)$ than for $\beta$-trace protein. The effect of a pathological decrease of CSF flow rate is of similar magnitude for the pathological increase of lumbar cystatin $\mathrm{C}$ concentration. Again, the slope for cystatin $\mathrm{C}$ is about $20 \%$ of that observed for $\beta$-trace protein. Another similarity in the dynamics of both these proteins is seen in cases of bacterial meningitis, where the pathological meningeal reaction leads to a decreased lumbar concentration of cystatin $\mathrm{C}$ like $\beta$-trace protein with about $60 \%$ of normal values and also with an invariance of the cystatin $\mathrm{C}$ concentration with decreasing CSF flow rate (data not shown).

\subsubsection{Transthyretin}

In contrast to NSE, S-100B, tau protein or $\beta$-trace protein for transthyretin, the blood-derived concentration is not negligible. With an 18 -fold larger concentration of liver-derived transthyretin in blood compared to CSF (Table 1), a small blood-derived fraction of about $10 \%$ can be calculated to contribute to transthyretin concentration in normal lumbar CSF (3\% in ventricular CSF) [1]. Transthyretin data for ventricular, cisternal or lumbar CSF have been re- 
ported earlier [32], indicating that there is a small decrease of concentration between ventricular and lumbar CSF and an increase of transthyretin concentrations with reduced CSF turnover below a spinal blockade(typically a restricted CSF flow rate). The data in Table 2 confirm this decrease between ventricular and lumbar CSF for the choroid plexus-derived (calculated) CSF fraction. The blood-derived fraction in CSF increases with increasing $Q_{\mathrm{Alb}}$ (Fig. $3 \mathrm{e})$. Using a mathematical correction for the bloodderived fraction [1], it becomes visible that the plexus-derived fraction is independent of changing $Q_{\mathrm{Alb}}$, like the concentration of the brain proteins S-100, NSE or tau protein.

\subsubsection{Biophysics of brain proteins in CSF}

The ventricular CSF concentration of a brain-derived protein depends on its tissue concentration, the molecular size-dependent diffusion into CSF and the CSF volume turnover rate (CSF flow rate). The rate of molecules diffusing through a border are characterized as molecular flux, J. Molecular flux is described by the general laws of diffusion (references in Ref. [2]), in particular Fick's first law: The rate of transfer ( $\mathrm{n}$ moles/s) of diffusing substance through unit area $\left(1 \mathrm{~cm}^{2}\right)$ of a section is proportional to the concentration gradient $(\mathrm{d} c / \mathrm{d} x)$ measured normal to the section, i.e.,

$J=-D \frac{\mathrm{d} c}{\mathrm{~d} x}\left[\mathrm{n}\right.$ moles $\left./ \mathrm{s} \mathrm{cm}^{2}\right]$

where $c$ is the concentration of diffusing substance, $x$ the space coordinate measured normal to the section, and $D$ is the diffusion coefficient. The concentration gradient $\mathrm{d} c / \mathrm{d} x$ is negative as its slope is negative in the direction of molecular flux. This function is a linear relation between molecular flux, $J$, and the local concentration gradient $\Delta c / \Delta x$.

The application of this net local concentration gradient to the border of CSF space has been shown earlier (Figs. 2 and 7 in Ref. [2]). With a constant concentration gradient, we get a constant molecular flux into CSF and in steady state with a constant CSF flow rate, we get a constant local CSF concentration. On this base, we can regard (a) the normal ventricle to lumbar (rostro-caudal) concentration gradient and (b) the pathological decrease of CSF flow rate, i.e., a blood-CSF barrier dysfunction in neurological diseases.

(a) The decrease of concentrations between ventricular and lumbar CSF for brain-derived proteins depends on the different tissue concentrations around CSF space and the corresponding local gradients.

Tau protein, NSE or S-100B, due to their neuronal or glial source must have higher concentrations in the tissue around the ventricular and cisternal CSF space than around the lumbar space in leptomeninges. The outside/in gradient $\Delta c$ in the range of ventricles is inverted to an inside/out gradient in lumbar CSF where the concentration in CSF (flowing down from ventricles) is larger than in the surrounding tissue (leptomeninges), with only a small local release of neuronal or glial proteins. This is different for the leptomeningeal proteins. The $\beta$-trace protein concentration is much higher in the leptomeninges than in the periventricular tissue, i.e., there is no inversion of the gradient $\Delta c$ downstream. These conditions are sufficient to explain the observed decreasing rostro-caudal concentration gradient of the tau protein group (3.5:1 to 1.5:1 in Table 2 ) and the increasing gradient for the $\beta$-trace protein group (1:11 to $1: 3.5$ in Table 2).

(b) In case of a pathological decrease in CSF volume $(\Delta \mathrm{Vol})$ turnover rate $(\Delta \mathrm{Vol} / \Delta t)$ and an independently steady release of brain cell-derived proteins via extracellular space into CSF, we get an increase of their concentrations $\mathrm{c}$ in $\mathrm{CSF}$ and subsequently a decrease of the gradient $\Delta c / \Delta x$. With a decreasing $\Delta c$, the molecular flux, $J_{\text {in }}$, is subsequently reduced, counteracting to some extent the effect of reduced CSF flow rate (increasing $c$ ). This effect can be regarded as a physical negative feedback loop, analogous to the metabolic inhibitory feedback loops in many biochemical processes. But nevertheless, with decreasing CSF turnover rate, the local concentration in CSF can still be increasing as long as a gradient $\Delta c>0$.

In the tau protein group, the constant concentration in lumbar CSF, invariant to decreasing CSF flow rate (Fig. $3 \mathrm{a}-\mathrm{c}$ ), is a consequence of the quantitatively compensating effect of increasing concentration $c$ (in ventricular CSF) and the subsequently increasing molecular outflux, $J_{\text {out }}$, along the subarachnoid space, in particular in the spinal canal, due to an increase of the local inside/out gradient 
$\Delta c / \Delta x$. Due to the linear correlation between increasing $c$ and increasing $\Delta c$, these effects compensate for each other quantitatively.

In contrast, the leptomeningeal proteins show an increasing lumbar CSF concentration with decreasing CSF flow rate (due to a non-inflammatory cause) (Fig. 3d). This can easily be explained by the steady release of $\beta$-trace protein into CSF according to the local outside/in gradients along the whole way downstream, which, together with the reduced volume turnover, must account for its increased CSF concentration.

The relative increase of CSF concentrations for $\beta$-trace protein with decreasing CSF flow rate is smaller in lumbar than in ventricular CSF as shown by the mean functions of the regression lines. In Fig. $3 \mathrm{~d}$, the linear regression line has the function:

$$
\begin{aligned}
& \beta \text {-trace protein }(\mathrm{L}-\mathrm{CSF}) \\
& \quad=14.8+0.31\left(Q_{\mathrm{Alb}} \times 10^{3}\right)(\mathrm{mg} / \mathrm{l}) .
\end{aligned}
$$

The corresponding function for the ventricular CSF is:

$$
\begin{aligned}
& \beta \text {-trace protein }(\mathrm{V}-\mathrm{CSF}) \\
& \quad=-0.5+0.90\left(Q_{\mathrm{Alb}} \times 10^{3}\right)(\mathrm{mg} / \mathrm{l}) .
\end{aligned}
$$

The data of ventricular CSF are from a group of patients $(n=13)$ without inflammatory processes but a blood-CSF barrier dysfunction (data not shown here). The intercept at $Q_{\mathrm{Alb}}=0$ in Eq. (3) is obtained from the empirical regression line and should be somewhat above zero. The corresponding mean concentration of normal V-CSF $(n=7)$ is $1.5 \mathrm{mg} / 1$ (Table 2) at mean $Q_{\mathrm{Alb}}=1.8 \times 10^{-3}$. The corresponding lumbar concentration is 11 -fold higher, with the intercept of 14.8, shown in Fig. $3 \mathrm{~d}$ and in Eq. (2).

This comparison of ventricular and lumbar CSF concentrations of $\beta$-trace protein with reference to the albumin quotient with different slopes $(0.90$ and $0.31)$ shows that the gradient, $\Delta c / \Delta x$, in ventricles must be steeper than in the lumbar region.

This linear relation between changing concentrations of brain-derived proteins and $Q_{\mathrm{Alb}}$ in the case of a reduced CSF flow rate is different from the hyperbolic relation of blood-derived proteins. In the case of the blood-derived proteins, any increase of
CSF concentration, e.g., due to reduced volume turnover, leads to an increase of the mean tissue concentration (Ref. [2] and references therein) with a non-linear increase of the local concentration gradient at the border with the CSF space. So, in fact, blood proteins show a positive feedback, i.e., with increasing CSF concentration the molecular flux into CSF is increasing. The corresponding hyperbolic concentration functions are shown in Fig. 2 for proteins of different size.

For brain-derived proteins, such an increase of the local concentration gradient between tissue and CSF as a consequence of increasing CSF concentration has no physical or physiological base. This would only be possible if an increasing CSF concentration could have a positive feedback on the brain cells, e.g., inducing an increased expression and/or increasing release of the brain proteins from corresponding brain cells. Such an unphysiological destabilization of the metabolic steady state of the cells has not been observed so far in any case.

These aspects can explain the origin of linear relations of brain-derived proteins versus nonlinear relations of blood-derived proteins with decreasing CSF flow rate (increasing $Q_{\text {Alb }}$ ).

\section{Discussion}

\subsection{CSF proteins from brain cells}

Proteins in CSF which originate from brain cells, like neurons or glial cells, which enter CSF primarily in the ventricular and cisternal spaces show the same dynamic:

- their concentration is decreasing between normal ventricular and lumbar CSF and

- their concentration does not vary with pathologically decreasing CSF flow rate, i.e., in cases of a so-called blood-CSF barrier dysfunction for blood-derived proteins.

The first observation, the decreasing rostro-caudal gradient, is easily explained by a net diffusion of neuronal and glial proteins from inside/out of CSF on the way downstream. This is due to the inversion 
of the tissue to CSF concentration gradient $\Delta c / \Delta x$ between the cisternal and lumbar CSF spaces (the leptomeningeal tissue has less of these cell types). This decreasing concentration of brain-derived proteins is opposite to the steady (non-linear) increase of the concentrations along the rostro-caudal flow way of blood-derived proteins in normal CSF.

The second observation, that protein concentrations in lumbar CSF do not change with decreasing CSF flow rate, i.e., increasing $Q_{\text {Alb }}$ (Fig. 3a-c) can be explained by derivation from laws of diffusion and CSF flow. The dynamic of brain proteins fit as a particular linear case into the molecular flux/CSF flow theory. With decreasing CSF flow rate, i.e., reduced CSF volume turnover, the ventricular CSF concentration is increasing linearly but with increasing CSF concentration there is downstream a larger concentration gradient for a larger molecular flux inside/out. Both effects compensate each other quantitatively, what keeps the lumbar CSF concentration constant.

In this way, the observed empirical data for NSE, S-100, tau protein or choroid plexus-derived transthyretin fraction are sufficiently explained by the theoretical model in which CSF flow rate represents the main modulator of CSF protein concentrations.

\subsection{CSF proteins from meningeal cells}

Proteins like $\beta$-trace protein or cystatin $C$, which originate primarily from leptomeningeal cells, show:

- An increasing concentration between normal ventricular and lumbar CSF.

- A linearly increasing concentration in CSF in case of pathologically decreasing CSF flow rate.

- A particular modification is observed in inflammatory meningeal processes.

The rostro-caudal increase of $\beta$-trace protein and cystatin C concentrations (Table 2) under normal conditions are easily understood as a steady release of proteins into CSF along its flow path, due to a local outside/in concentration gradient at the border with the subarachnoid space. The $\beta$-trace protein concentration is increasing from $1.5 \mathrm{mg} / 1$ in the ventricles to $16.6 \mathrm{mg} / \mathrm{l}$ in the lumbar subarachnoid space. This larger concentration in lumbar CSF has consequences for the dynamics of $\beta$-trace protein in the case of a pathologically reduced CSF flow rate. In Eqs. (2) and (3), the smaller slope for lumbar CSF indicates that the local gradient $(\Delta c / \Delta x)$ between tissue and CSF space is larger in the ventricles than in the lumbar subarachnoid space. The 11-fold increase of $\beta$-trace protein concentration between ventricular and lumbar CSF is not related to a correspondingly 11 -fold higher release of $\beta$-trace protein in the leptomeninges compared to the extra-ventricular tissue.

This second observation, the linear increase of $\beta$-trace protein concentration in lumbar CSF in case of pathologically decreasing CSF flow rate, can be important also from a theoretical point of view. This linear increase, e.g., of $\beta$-trace protein or cystatin $C$ concentration, is different from the non-linear hyperbolic function [2] for blood-derived proteins in CSF (Fig. 2).

The type of positive (physical) feed back loop for blood-derived proteins is missing in the case of the cell metabolism-dependent release of proteins $(\beta$ trace protein, cystatin $\mathrm{C}$, etc.) into CSF; i.e., an increasing CSF concentration of leptomeningeal proteins in CSF does not induce an increased release of these proteins from brain cells. Such a process of spontaneous induction and increased expression of proteins (analogous to an autocatalytic process) has not been observed so far. This explains why the dynamic of these proteins can be described by Fick's first law with a linear relation between molecular flux and concentration gradient. As an interesting support of the relevance of CSF flow for brain-derived proteins, the report of Grubb and Löfberg [27] shows for cystatin $\mathrm{C}$ an age-related CSF concentration, decreasing shortly after birth [27]. This is consistent with the age-related increase of CSF flow rate $[2,6]$ due to the maturation of arachnoid villi at the time of birth and shortly after: A maximum CSF flow rate is reached about 4 months after birth with the correspondingly lowest CSF protein concentration, slowly increasing again over the next decades due to a decreasing CSF production rate [12,37]. This similarity of the normal age-related dynamics between meningeal proteins (linear increase) and 
blood-derived proteins (hyperbolic increase) gives further evidence for the relevance of CSF flow rate.

These findings from pathology confirm that the molecular flux/CSF flow theory represents a complete conceptual framework for explaining the dynamics of both the blood- and brain-derived proteins in CSF. To support the validity of the theory, we point to its interpretative power in making sense of unusual observations. This is shown in three examples below.

(1) A particular example is represented by the dynamic of $\beta$-trace protein in the case of bacterial or tuberculous meningitis. In these cases, $\beta$-trace protein values in lumbar CSF are below normal concentrations but remain constant, i.e., independent of the decreasing CSF flow rate [36] (Fig. 3d). The observed data could be explained as a reduced release of $\beta$-trace protein along the lumbar meningeal region in the case of bacterial meningitis. In fact, the CSF concentrations, lower than normal lumbar CSF concentrations, represent the lower concentrations typical for cisternal CSF. With the knowledge that in bacterial meningitis the meningeal release of $\beta$-trace protein is inhibited, the measured protein concentration in lumbar CSF would originate primarily from tissue around the ventricular and cisternal area, like NSE, S-100 or tau protein. In this sense, the $\beta$-trace protein concentration in these inflammatory meningeal processes follows the rules for the brain cell-derived proteins with an invariance to changes of CSF flow rate (Fig. 3d) in contrast to the non-inflammatory reduction of CSF flow rate (Fig. 3d). These aspects of $\beta$-trace protein (and cystatin $\mathrm{C}$ ) raise new questions for the investigation of leptomeningeal processes in a bacterial meningitis, a disease which still has the highest fatal outcome amongst inflammatory neurological diseases.

(2) The normal CSF concentration of the soluble cell adhesion molecule, s-ICAM, originates with more than $70 \%$ from blood [33] and the s-ICAM $\mathrm{CSF} /$ serum quotient should follow a hyperbolic function with increasing albumin quotient as shown for other blood-derived proteins (Fig. 2). In fact, the quotient, $Q_{\text {ICAM }}$, approaches a limiting value like a saturation curve (Fig. 3f). From molecular flux /CSF flow theory, we understand that there must be, in addition to diffusion, an influence involved in the pathomechanism, which is counteracting a steady increase of concentration. Such a type of reaction is observed in negative feed back loops, common to many biochemical regulation processes. So in this case, we must propose either a faster s-ICAM degradation or an elimination process with increasing s-ICAM concentration. This is typical for biochemically controlled substances, a condition which has been shown to be fulfilled for upregulation of sICAM biosynthesis, too [34]. This leads to the understanding that s-ICAM is not inert to biochemical reactions and further specific investigations regarding s-ICAM control mechanisms are needed. This result is very different from the approach proposed on the basis of an earlier, unfounded barrier model [38], which conflicts with established observations on blood-CSF barrier dysfunction.

(3) Evaluation of transthyretin in CSF, about $90 \%$ of which originates from the choroid plexus, represents another intelligible application of the molecular flux/CSF flow theory. As shown earlier [1] by a mathematical approach, it is possible to subtract the calculated blood-derived fraction of transthyretin from the total concentration in CSF. In this way, we can demonstrate that the choroid plexus-derived fraction behaves like NSE, or S-100, or tau protein: it remains independent of CSF flow rate (Fig. 3e).

So, finally we get a consistent concept for the evaluation of brain-derived and blood-derived proteins or proteins with mixed sources in the brain, leptomeninges or blood. This concept shows that it is important for evaluation of some brain proteins in CSF to refer to the albumin quotient as a measure of CSF flow rate. The lack of a $Q_{\mathrm{Alb}}$-related evaluation of ACE [1], s-ICAM [33], or $\beta$-trace protein (Fig. 3d) may lead to erroneous interpretations of an increased (or decreased) protein concentration in lumbar CSF. In initial investigations to introduce a new brain protein into diagnostic approaches, the detection of the ventricular to lumbar concentration gradient in addition to the CSF/blood gradient can give important information about the source of a protein and its pathophysiological processes. This basic information helps also to decide whether the absolute concentration (Fig. 3a-d, cases of dominantly brainderived proteins) or the $\mathrm{CSF} /$ serum concentration quotient (Fig. 3e and $\mathrm{f}$, cases of dominantly bloodderived proteins) should be used to produce the most sensitive evaluation. This evaluation concept for brain 
proteins completes the evaluation programs for intrathecal synthesis of blood-derived proteins in $\mathrm{CSF} /$ serum quotient diagrams [4-6].

It seems beyond any doubt that CSF turnover is the crucial determinant for the concentration of its macromolecular components. The empirical data and their interpretations for clinical purposes refer to a mean turnover rate, which does not need to take into account the local and temporal fluctuations, including to-and-fro motions of the CSF by cardiac cycles $[9,10,40,41]$ or the circadian rhythms (references in Ref. [9]). The usually reported aqueductal CSF velocities can be very fast due to an aqueduct stenosis [9]. But, the absolute values of aqueductal velocities cannot be regarded as representative for the overall CSF turnover rate. This is a limitation of imaging techniques for a correlation with the disease-related change of the albumin quotient.

\section{References}

[1] Reiber H. CSF flow-its influence on CSF concentration of brain-derived and blood-derived proteins. In: Teelken A, Korf J, editors. Neurochemistry. New York: Plenum 1997: 51-72.

[2] Reiber H. Flow rate of cerebrospinal fluid (CSF) - a concept common to normal blood-CSF barrier function and to dysfunction in neurological diseases. J Neurol Sci 1994;122: 189-203.

[3] Reiber H. Biophysics of protein diffusion from blood into CSF: the modulation by CSF flow rate. In: Greenwood J, Begley D, Segal M, editors. New concepts of a blood-brain barrier. New York: Plenum; 1995 p. 219-27.

[4] Reiber H. Die diagnostische Bedeutung neuroimmunologischer Reaktionsmuster im Liquor cerebrospinalis. Lab Med 1995;19:444-62.

[5] Reiber H. Cerebrospinal fluid-physiology, analysis and interpretation of protein patterns for diagnosis of neurological diseases. Mult Scler 1998;4:99-107.

[6] Reiber H, Peter JB. Cerebrospinal fluid analysis: disease-related data patterns and evaluation programs. J Neurol Sci 2001;184:101-22.

[7] Herndon RM, Brumback RA. The cerebrospinal fluid. Boston: Kluwer Academic Publishing; 1989.

[8] Davson H. Physiology of the ocular and cerebrospinal fluids. London: Churchill; 1956.

[9] Parkkola RK, Komu MES, Äärimaa TM, Alanen MS, Thomsen C. Cerebrospinal fluid flow in children with normal and dilated ventricles studied by MR imaging. Acta Radiol 2001;42:33-8.

[10] Greitz D, Hannerz J. A proposed model of cerebrospinal fluid circulation: observations with radionuclide cisternography. Am J Neuroradiol 1996;17:431-8.

[11] Rapoport SI. Passage of proteins from blood to cerebrospinal fluid. In: Wood JH, editor. Neurobiology of cerebrospinal fluid, vol. 2. New York: Plenum; 1983 p. 233-45.

[12] Rubenstein E. Relationship of senescence of cerebrospinal fluid circulatory system to dementia of the aged. Lancet 1998;351:283-5.

[13] Dorta Contreras AJ. Reibergramas: elemento esencial en el analisas inmunologico del líquido cefalorraquídeo. Rev Neurol 1999;28:996-8.

[14] Thompson EJ. The CSF proteins: a biochemical approach. Amsterdam: Elservier; 1988.

[15] Andreasen N, Vanmechelen E, Van de Voorde A, et al. Cerebrospinal fluid tau protein as a biochemical marker for Alzheimer's disease: a community based follow up study. J Neurol, Neurosurg Psychiatry 1998;64:298-305.

[16] Hulstaert F, Blennow K, Ivanoiu A, et al. Improved discrimination of $\mathrm{AD}$ patients using $\beta$-amyloid (1-42) and tau levels in CSF. Neurology 1999;52:1555-62.

[17] Zerr I, Bodemer M, Räcker S, et al. Cerebrospinal fluid concentration of neuron-specific enolase in diagnosis of Creutzfeldt-Jakob disease. Lancet 1995;345:1609-10.

[18] Otto M, Stein H, Szudra A. S-100 protein concentration in the cerebrospinal fluid of patients with Creutzfeldt-Jakob disease. J Neurol 1997;244:566-70.

[19] van Engelen BGM, Lamers KJB, Gabreels FJM, Wevers RA, van Geel WJA, Borm GF. Age-related changes of neuronspecific enolase, S-100 protein and myelin basic protein concentrations in CSF. Clin Chem 1992;38:813-6.

[20] Jacobi C, Reiber H. Clinical relevance of increased neuronspecific enolase concentration in cerebrospinal fluid. Clin Chim Acta 1988;177:49-54.

[21] Schaarschmidt H, Prange H, Reiber H. Neuron-specific enolase concentrations in blood as a prognostic parameter in cerebrovascular diseases. Stroke 1994;24:558-65.

[22] Sindic CJM, Chalon M, Cambiaso C, Laterre E, Masson P. Assessment of damage to central nervous system by determination of S-100 protein in the cerebrospinal fluid. J Neurol, Neurosurg Psychiatry 1982;45:1130-6.

[23] Hoffmann A, Conradt HS, Gross G, Nimtz M, Lottspeich F, Wurster U. Purification and chemical characterization of $\beta$-trace protein from human cerebrospinal fluid: its identification as prostaglandin D synthase. J Neurochem 1993;61:4516.

[24] Urade Y, Katahama K, Ohishi J, Kaneko T, Mizuno N, Hayaishi O. Dominant expression of m-RNA for prostaglandin D-synthetase in leptomeninges, choroid plexus and oligodendrocytes of the adult rat brain. Proc Natl Acad Sci 1993;90:9070-4.

[25] Blödorn B, Mäder M, Urade Y, Hayaishi O, Felgenhauer K, Brück W. Choroid plexus: the major site of mRNA expression for the $\beta$-trace protein (prostaglandin D synthetase) in human brain. Neurosci Lett 1996;209:117-20.

[26] Tumani H, Nau R, Felgenhauer K. $\beta$-trace protein in cerebrospinal fluid: a blood-CSF barrier-related evaluation in neurological diseases. Ann Neurol 1998;44:882-9. 
[27] Grubb A, Löfberg H. Human gamma-trace. Structure, function and clinical use of concentration measurements. Scand J Clin Lab Invest, Suppl 1985;177:7-13.

[28] Cole T, Dickson PW, Esnard F, et al. The cDNA structure and expression analysis of the genes for the cysteine proteinase inhibitor cystatin $\mathrm{C}$ and for $\beta 2$-microglobulin in rat brain. Eur J Biochem 1986;186:35-42.

[29] Ohe Y, Ishikawa K, Itoh Z, Tatemoto K. Cultured leptomeningeal cells secrete cerebrospinal fluid proteins. J Neurochem 1996;67:964-71.

[30] Wu D, Schreiber G. Expression of retinol-binding protein in RNA in mammalian choroid plexus. Comp Biochem Physiol 1992;101B:99-406.

[31] Herbert J, Wilcox JN, Pham K-TC, et al. Transthyretin: a choroid plexus-specific transport protein in human brain. Neurology 1986;36:900-11.

[32] Weisner B, Roethig H-J. The concentration of prealbumin in cerebrospinal fluid, indicator of CSF circulation disorders. Eur Neurol 1983;22:96-105.

[33] Lewczuk P, Reiber H, Tumani H. Intercellular adhesion molecule-1 (sICAM-1) in cerebrospinal fluid-the evaluation of blood-derived and brain-derived fractions in neurological diseases. J Neuroimmunol 1998;87:156-61.

[34] Lewczuk P, Reiber H, Korenke GC, Bollensen E, Dorta-Contreras AJ. Intrathecal release of sICAM-1 into CSF in neu- roborreliosis-increased brain-derived fraction. J Neuroimmunol 2000;103:93-6.

[35] Lewczuk P, Reiber H, Ehrenreich H. Prothrombin in normal human cerebrospinal fluid originates from the blood. Neurochem Res 1998;23:1027-30.

[36] Tumani H, Reiber H, Nau R, et al. Beta-trace protein concentration in cerebrospinal fluid is decreased in patients with bacterial meningitis. Neurosci Lett 1998;242:5-8.

[37] May C, Kays SA, Attack JR, Schapiro MB, Friedland RP, Rapopport SI. Cerebrospinal fluid production is reduced in healthy aging. Neurology 1990;40:500-3.

[38] Rieckmann P, Nünke K, Burchhardt M, et al. Soluble intercellular adhesion molecule-1 in cerebrospinal fluid: an indicator for the inflammatory impairment of the blood-cerebrospinal fluid barrier. J Neuroimmunol 1993;47:133-40.

[39] Reiber H, Felgenhauer K. Protein transfer at the blood-CSF barrier and the quantitation of the humoral immune response within the central nervous system. Clin Chim Acta 1987; 163:319-28.

[40] Ridgway JP, Turnbull LW, Smith MA. Demonstration of pulsatile cerebrospinal fluid flow using magnetic resonance phase imaging. Br J Radiol 1987;60:423-7.

[41] Klose U, Strik C, Kiefer C, Grodd W. Detection of a relation between respiration and CSF pulsation with an echoplanar technique. J Magn Reson Imaging 2000;11:438-44. 